\title{
CARDIAC BIOMARKERS and CEREBRAL ISCHAEMIA
}

Fiori Patrizia, Corbo Antonio, Iorillo Luigi, Savino Patrizia, Bellizzi Gennaro, Dragonetti Carmela, Giannetti Luigi Maria, Mazza Emerico, Tammaro Carmine, Monaco Antonio

S. Ottone Frangipane Hospital, ASL AV, Ariano irpino (AV) - University of Naples

\section{Introduction}

Troponin ths (Tro ths) and N-Terminal-pro-Brain Natriuretic peptide (NT-pro-BNP) are increased in cerebrovascular conditions, especially in class III/IV, C/D New York Heart Association and American Cardiology Association scales. The aim of our study was to assess the relation with the involved cerebral region.

\section{Materials and Methods}

So far, we recruited 552 acute ischaemic stroke (AS, age 78,6 sd 11,5). They underwent blood withdrawals at day 1,3 and 7. Computerized Tomography and/or Magnetic Resonance Imaging were performed at admission, after 24 hours and, if necessary, repeated later.

\section{Results}

No significant differences of cardiac biomarkers were related to the region of the AS (Internal Carotid Artery, Middle Cerebral Artery, Anterior Cerebral Artery AS: Tro ths 73,4 sd $430,2 \mathrm{pg} / \mathrm{ml}$, NT-pro-BNP 3898,6 sd 6648,4 pg/ml; VertebroBasilar AS: Tro ths 60 sd 153,5 pg/ml; NT-proBNP: 3607,9 sd 6143,7 pg/ml, p ns).
These results were confirmed in subgroup analysis among cardiac biomarkers in fronto-parietal (FP: Tro ths 24,93 sd 19,37 pg/ml; NT-pro-BNP 1717,1 sd 1996,4 pg/ml), temporo-insular (TI: Tro ths 23,9 sd 15,1 pg/ml; NT-pro-BNP 2581 sd 4166,1 pg/ml), capsulo-nuclear (CN: Tro ths 27,73 sd 27,27 pg/ml; NT-pro-BNP 2022,4 sd 2435,5 pg/ml), occipital (O: Tro ths 41,68 sd 42,57 pg/ml; NT-pro-BNP 2398,8 sd 2198,4 pg/ml), cerebellar-brainstem (CB: Tro ths 30,6 sd 26,4 pg/ml; NT-pro-BNP 2080,8 sd 2771,9 $\mathrm{pg} / \mathrm{ml}$ ) AS (Fig. 1a and 1b). Levels of cardiac biomarkers, as the extension of cerebral ischaemia, were related to the severity of cardiac dysfunction, responsible of diffuse hypoxic encephalopathy in severe cases.

\section{Conclusions}

Our data highlight the importance of cardiological stratification for assessing the risk of reduced perfusion and increased diffusion-restricted cerebral tissue in AS.

\begin{tabular}{|l|c|c|c|c|c|}
\hline & FP & TI & CN & 0 & CB \\
\hline Tro ths (pg/ml) & $24,93 \mathrm{sd} \mathrm{19,37}$ & $23,9 \mathrm{sd} 15,1$ & $27,73 \mathrm{sd} 27,27$ & $41,68 \mathrm{sd} \mathrm{42,57}$ & $30,6 \mathrm{sd} 26,4$ \\
\hline NT-pro-BNP (pg/ml) & $1717,1 \mathrm{sd} 1996,4$ & $2581 \mathrm{sd} 4166,1$ & $2022,4 \mathrm{sd} 2435,5$ & $2398,8 \mathrm{sd} 2198,4$ & $2080,8 \mathrm{sd} 2771,9$ \\
\hline
\end{tabular}
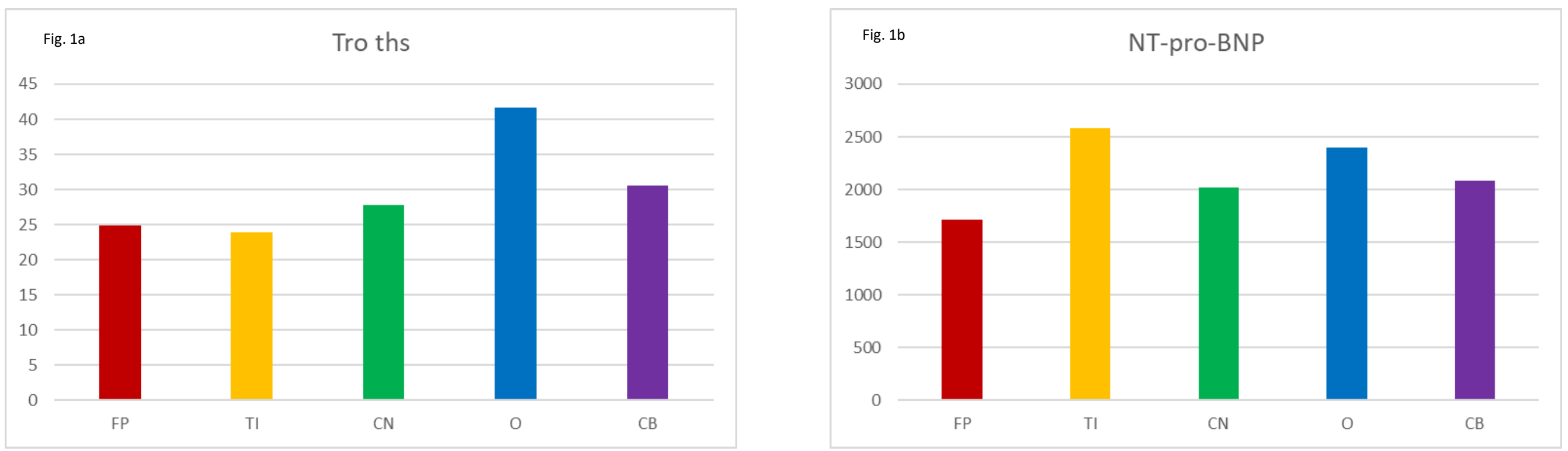